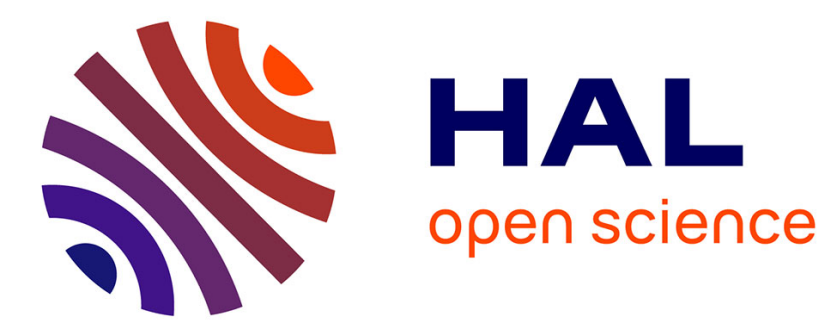

\title{
Effets des apprentissages de grande section de maternelle et des pratiques familiales en lien avec la scolarité sur les acquisitions en fin de CE1
}

Youssef Tazouti, Annette Jarlégan

\section{- To cite this version:}

Youssef Tazouti, Annette Jarlégan. Effets des apprentissages de grande section de maternelle et des pratiques familiales en lien avec la scolarité sur les acquisitions en fin de CE1. Enfance, 2013, 2013 (02), pp.139 - 157. 10.4074/S0013754513002036 . hal-01437145

\section{HAL Id: hal-01437145 \\ https://hal.univ-lorraine.fr/hal-01437145}

Submitted on 17 Jan 2017

HAL is a multi-disciplinary open access archive for the deposit and dissemination of scientific research documents, whether they are published or not. The documents may come from teaching and research institutions in France or abroad, or from public or private research centers.
L'archive ouverte pluridisciplinaire HAL, est destinée au dépôt et à la diffusion de documents scientifiques de niveau recherche, publiés ou non, émanant des établissements d'enseignement et de recherche français ou étrangers, des laboratoires publics ou privés. 


\section{Enfance}

http://www.necplus.eu/ENF

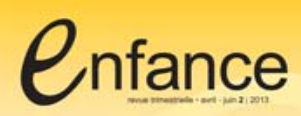

Additional services for Enfance:

Email alerts: $\underline{\text { Click here }}$

Subscriptions: $\underline{\text { Click here }}$

Commercial reprints: $\underline{\text { Click here }}$

Terms of use : $\underline{\text { Click here }}$

\section{Effets des apprentissages de grande section de maternelle et des pratiques familiales en lien avec la scolarité sur les acquisitions en fin de CE1}

Youssef Tazouti et Annette Jarlégan

Enfance / Volume 2013 / Issue 02 / June 2013, pp 139 - 157

DOI: 10.4074/S0013754513002036, Published online: 15 July 2013

Link to this article: http://www.necplus.eu/abstract_S0013754513002036

How to cite this article:

Youssef Tazouti et Annette Jarlégan (2013). Effets des apprentissages de grande section de maternelle et des pratiques familiales en lien avec la scolarité sur les acquisitions en fin de CE1. Enfance, 2013, pp 139-157 doi:10.4074/

S0013754513002036

Request Permissions : $\underline{\text { Click here }}$ 


\title{
Effets des apprentissages de grande section de maternelle et des pratiques familiales en lien avec la scolarité sur les acquisitions en fin de CE1
}

\author{
Youssef TAZOUTI ${ }^{1}$ et Annette JARLÉGAN ${ }^{2}$
}

\begin{abstract}
RÉSUMÉ
Cette étude examine les effets a long terme des pratiques éducatives familiales en lien avec la scolarité et des apprentissages effectués en grande section de maternelle sur les acquisitions de fin de CE1. La recherche porte sur une cohorte de 129 enfants évalués à deux reprises. Durant l'année de GS, des épreuves d'évaluation des apprentissages premiers ont été administrées aux élèves dans 7 écoles maternelles. Deux ans plus tard, les mêmes élèves ont été évalués collectivement par des épreuves de français et de mathématiques. Par ailleurs, des questionnaires ont été proposés aux parents afin d'opérationnaliser certaines dimensions de leurs pratiques éducatives. Plusieurs modèles de régression multiple ont été estimés pour apprécier l'effet des différentes variables de l'étude sur les performances scolaires en CE1. Les résultats mettent en évidence des liens forts entre certaines acquisitions de GS (par ex. discrimination auditive, organisation spatiale, dénombrement) et les apprentissages en français et en mathématique en CE1. De plus, des liens sont constatés entre les pratiques parentales familiarisant l'enfant aux fonctions de l'écrit et les apprentissages scolaires.

MOTS CLÉS : ÉCOLE MATERNELLE, APPRENTISSAGES PREMIERS, PERFORMANCES SCOLAIRES, FONCTION MÉTACOGNITIVE, PRATIQUES ÉDUCATIVES FAMILIALES.
\end{abstract}

${ }^{1}$ Université de Lorraine, InterPsy - EA 4165, Nancy F-54528, IUFM de Lorraine, 5 rue Paul Richard, C.O. 3 Maxéville, 54528 Laxou Cedex. Email : youssef.tazouti@univ-lorraine.fr

${ }^{2}$ Université de Lorraine, LISEC- EA 2310, Nancy F-54015, Université de Lorraine (Nancy 2), Sciences de l'Éducation, 23 boulevard Albert 1 ${ }^{\mathrm{er}}$, BP 3397, 54015 Nancy Cedex. Email : annette.jarlegan@univ-lorraine.fr 


\section{ABSTRACT \\ The effects of learning at kindergarten and family educational practices on French and math skills at the end of second grade}

The present research examines the long-term effects of learning at kindergarten and families' educational practices on French and math skills at the end of second grade. Our study was based on a cohort of 129 children, who were evaluated on two occasions. During the kindergarten year of pre-school, evaluation tests were administered to students in seven classes. Two years later, the same children were evaluated collectively in French and math. In addition, parents were asked to complete questionnaires assessing certain dimensions of their educational practices. We used multiple regression models to evaluate the effects of the study variables on academic performance in second grade. The results revealed strong links between certain skills learned at kindergarten (par ex., auditory discrimination, spatial organization, counting) and French and math skills in second grade. We also found links between parental practices familiarizing children with writing functions and academic achievement.

KEY-WORDS: KINDERGARTEN, EARLY LEARNING, ACADEMIC ACHIEVEMENT, FAMILY EDUCATIONAL PRACTICES. 


\section{INTRODUCTION}

En 2006, l'OCDE a souligné la grande diversité des institutions qui accueillent les jeunes enfants de par le monde et la diversité de leurs objectifs. À titre d'exemple, en Europe, on distingue deux courants dominants. Le premier correspondant à l'approche " préprimaire » des pays latins et anglophones consiste à proposer aux enfants des situations d'apprentissage structurées et progressives visant à les préparer explicitement à l'école élémentaire. Le second qui correspond à la tradition de la " pédagogie sociale » que l'on retrouve principalement en Europe du Nord et dans les pays germaniques met plus l'accent sur la socialisation et sur l'exploitation des situations d'apprentissage issues de la vie en groupe. Toutefois, malgré la diversité des institutions, des modèles et de leurs orientations pédagogiques, l'enseignement préscolaire a partout pour vocation de socialiser les enfants mais aussi de leur permettre de construire les habiletés de bases dont ils auront besoin pour réussir à l'école ultérieurement. Parmi les apprentissages premiers qui sont visés, on trouve les compétences en littératie précoce qui correspondent à l'ensemble des connaissances et des habiletés nécessaires aux futurs apprentissages concernant la lecture et l'écriture (Whitehurst \& Lonigan, 1998 ; 2001). Hatcher, Hulme et Snowling (2004) ont identifié trois dimensions de la littératie précoce : le vocabulaire, la connaissance des lettres et la conscience phonologique. On trouve également les compétences mathématiques premières, comme la compréhension du nombre, la reconnaissance des formes et la résolution de problèmes logiques, qui aideront les enfants à acquérir les apprentissages mathématiques ultérieurs.

De nombreuses méta-analyses soulignent l'importance de la fréquentation d'une structure de pré-scolarisation pour la scolarité ultérieure. Elles montrent que les programmes de préscolarisation ont un impact positif sur le développement et les apprentissages ultérieurs des enfants (Goldring \& Presbrey, 1986 ; Hattie, 2009). Il ressort notamment de ces travaux que les programmes les plus efficaces sont ceux qui sont les plus structurés et également ceux qui sont gérés par des personnels bien formés (Innocenti \& White, 1993). Par ailleurs, Fusaro (1997) montre que les enfants qui fréquentent les structures préscolaires une journée complète ont des résultats significativement supérieurs à ceux qui les fréquentent seulement une demi-journée. De plus, Jones (2002) constate que la fréquentation de la maternelle toute la journée a des effets positifs différés sur les apprentissages scolaires au début de la scolarité élémentaire. L'auteur constate également un impact plus grand sur l'apprentissage de la lecture que sur les acquisitions mathématiques. Cet effet différé est confirmé par La Paro et Pianta (2000) qui trouvent aussi des liens significatifs entre les apprentissages en maternelle et les résultats des élèves à l'issue des deux premières années de l'enseignement obligatoire. Il est également intéressant de souligner que les enfants qui fréquentent les structures préscolaires développent non seulement plus d'aptitudes cognitives que ceux qui restent au domicile familial mais qu'ils acquièrent aussi plus d'aptitudes sociales et émotionnelles que ces derniers (Applegate, 1986). Selon certaines études (Nelson, Westhues, \& MacLeod, 
2003), ces effets bénéfiques de la préscolarisation n’apparaissent que si l'enfant fréquente les programmes de préscolarisation au moins un an. Enfin, d'autres études ont mis en évidence une efficacité différentielle de ces programmes de préscolarisation : les élèves appartenant à des catégories socioprofessionnelles défavorisées ou à des minorités ethniques en tirent un bénéfice plus important que les autres (Collins, 1984 ; Goldring \& Presbrey, 1986 ; Harrell, 1983 ; Nelson, Westhues, \& MacLeod, 2003).

Même si les compétences en littératie précoce et les compétences mathématiques de base s'acquièrent essentiellement à l'école, le rôle de l'environnement familial de l'enfant n'est pas à négliger dans ces apprentissages. En effet, d'une part, les enfants entrent à l'école avec des habiletés langagières diverses, en lien avec le milieu familial, et ces différences jouent un rôle important lors du passage de l'oral à l'écrit (Murray, Stahl, \& Ivey, 1996 ; Purcell-Gates, 1996). D'autre part, de nombreux travaux montrent que les relations entre parents et enseignants ont un effet sur les apprentissages des enfants (Bronfenbrenner 2005 ; Epstein 2001 ; Hoover-Dempsey \& Sandler, 2005) et que l'implication des parents dans la scolarité des enfants a un impact positif sur leurs performances scolaires tout au long de la scolarité primaire et secondaire (Hoover-Dempsey, Battiato, Walker, Reed, DeJong, \& Jones, 2001 ; Fan \& Chen, 2001 ; Izzo, Weissberg, Kasprow, \& Fendrich, 1999 ; Rosenzweig, 2000). Cette implication parentale a son importance dès les premières années de l'enfance (Fantuzzo et al. 2004 ; Manz et al. 2004 ; McWayne et al. 2004). Tazouti, Viriot-Goeldel, Matter, Geiger-Jaillet, Carol et Deviterne (2011) ont ainsi montré que certaines pratiques éducatives en relation avec la préscolarisation de l'enfant ont un impact sur les apprentissages des élèves en maternelle. Par exemple, des pratiques diversifiées et fonctionnelles dans le domaine de l'écrit (par ex. lecture de livres de jeunesse, fréquentation de bibliothèques...) sont plus corrélées avec les performances que d'autres, plus traditionnelles et formelles (par ex. enseignement des correspondances grapho-phonémiques).

En France, l'école maternelle, avec son personnel formé, ses programmes scolaires nationaux, ses activités structurées et sa fréquentation assidue, a été longtemps considérée comme le maillon fort du système éducatif français. Toutefois, au vu des difficultés repérées chez un nombre non négligeable d'enfants à l'entrée du CP, le Haut Conseil de l'Education, dans son rapport de 2007, s'est interrogé sur la capacité de l'école maternelle à réduire les inégalités sociales et à mettre les enfants dans les meilleures dispositions pour effectuer les apprentissages ultérieurs. Jusqu'à présent, les travaux français portant sur l'école maternelle se sont essentiellement attachés à en décrire le fonctionnement (par ex. Cèbe \& Paour, 2000 ; Paour, Cèbe \& Haywood, 2000) ou à en évaluer l'impact sur les acquisitions immédiates des enfants (par ex. Caille \& Rosenwald, 2006 ; Morlaix \& Suchaut, 2007). À notre connaissance, peu d'études se sont intéressées à l'impact différé des apprentissages premiers effectués en école maternelle et de l'implication parentale précoce sur les apprentissages ultérieurs, malgré leur possible importance cruciale pour la suite de la scolarité. 
L'objectif de la recherche que nous présentons ici est précisément d'étudier ces effets différés. Il s'agira d'une part, d'étudier les effets des apprentissages premiers effectués à l'école maternelle et, d'autre part, d'évaluer les effets des pratiques éducatives fa miliales en lien avec la scolarité sur les apprentissages de fin de CE1.

\section{MÉTHOde}

\section{Participants}

Cette recherche a porté sur une cohorte de 129 enfants suivie de la grande section de maternelle à la fin du CE1. L'échantillon des élèves est composé de 78 filles et 51 garçons. Parmi les parents 89 sont de nationalité française et $11 \%$ de nationalité étrangère. Les enfants étaient âgés en moyenne de 5 ans 7 mois (écart-type de 3 mois) en grande section. En CE1, ils étaient âgés en moyenne de 7 ans 8 mois (écart-type de 5 mois). L'échantillon des écoles est composé de sept écoles maternelles et de sept écoles élémentaires contrastées sur le plan socioéconomique. Deux écoles maternelles et deux écoles élémentaires accueillaient majoritairement des enfants des milieux populaires. Les autres écoles scolarisaient des enfants des milieux moyens et favorisés.

\section{Procédure}

Des épreuves d'évaluation des apprentissages premiers ont été administrées aux élèves en fin d'année de GS dans 7 écoles maternelles. Deux ans plus tard, les mêmes élèves ont été évalués collectivement par des épreuves de français et de mathématiques. La passation des différentes épreuves a été effectuée en petits groupes de six élèves en maternelle et par classe entière au CE1. La durée de passation n'excédait pas 40 minutes dans les deux cas. Par ailleurs, un questionnaire a été proposé aux parents afin d'opérationnaliser leurs pratiques éducatives.

\section{Mesures}

\section{Appartenance sociale}

L'appartenance sociale des familles a été opérationnalisée par le calcul d'un indice économico-culturel agrégeant trois indicateurs : 1 . le niveau d'instruction de la mère ; 2. le niveau d'instruction du père ; 3. l'espace disponible du logement, à savoir le nombre de pièces divisé par le nombre d'habitants. Les corrélations modérées à élevées entre les trois indicateurs (elles varient entre 0,25 et 0,70 et sont toutes significatives à $\mathrm{p}<0.01)$ justifient leur regroupement. Cet indice économico-culturel présente une moyenne de 2,74 et un écart-type de 0,71 .

\section{Apprentissages premiers en GS}

Les performances des enfants ont été mesurées dans neuf domaines relatifs aux apprentissages mathématiques (organisation spatiale, dénombrement, rythmes), à ceux liés à la lecture-écriture (discrimination visuelle, discrimination 
auditive, correspondances oral/écrit) ainsi qu'à des apprentissages transversaux (graphisme, mémoire visuelle, mémoire auditive). Les épreuves ont été construites en s'inspirant d'outils évaluant des compétences intervenant dans l'acquisition de la lecture, de l'écriture et du calcul (Tazouti et al. 2011) : l'échelle NBA1-T (Ravard \& Rabreau, 2005) et les échelles des évaluations nationales Grande Section de Maternelle (MEN, 2003). Ces épreuves ne prétendent pas rendre compte de l'ensemble des compétences acquises par l'enfant au seuil de l'école élémentaire, mais en pointer quelques-unes parmi les plus déterminantes pour la poursuite des apprentissages. Pour des exemples d'items nous renvoyons le lecteur à l'annexe 1 .

Le tableau 1 fournit des statistiques descriptives des résultats aux neuf épreuves relatives aux apprentissages premiers. On constate que les épreuves de graphisme et de discrimination auditive présentent les moyennes les plus faibles (respectivement 0,41 et 0,55 ). En revanche, c'est l'épreuve de dénombrement qui présente la moyenne la plus élevée $(0,86)$.

\section{Performances scolaires en CE1}

Les performances scolaires des élèves ont été mesurées en français et en mathématiques en fin d'année par des épreuves standardisées construites à partir des évaluations nationales (MEN, 2003). En français, quatre exercices évaluant la compréhension du texte et la maîtrise des outils de la langue ont été administrés aux élèves. En mathématiques, les exercices évaluaient les domaines de la numération, de la géométrie et de la résolution de problèmes. Ces différentes épreuves ont été choisies car elles correspondent aux compétences qui doivent être acquises par l'enfant en fin de CE1. Le tableau 1 fournit le nombre d'items et les coefficients de consistance interne (alpha de Cronbach) des épreuves de mathématiques et de français. Ils sont satisfaisants (respectivement 0,84 et 0,85 ).

\section{Tableau 1.}

Statistiques descriptives des épreuves de l'échelle d'évaluation des apprentissages premiers en GS et des performances scolaires en CE1.

\begin{tabular}{lcccc}
\hline & Nombre d'items & Moyenne & Écart type & Erreur type \\
\hline Graphisme & 4 & 0,41 & 0,25 & 0,02 \\
Organisation spatiale & 4 & 0,78 & 0,26 & 0,02 \\
Rythme & 3 & 0,77 & 0,30 & 0,03 \\
Discrimination visuelle & 3 & 0,85 & 0,27 & 0,02 \\
Discrimination auditive & 4 & 0,55 & 0,33 & 0,02 \\
Mémoire visuelle & 6 & 0,83 & 0,23 & 0,02 \\
Mémoire auditive & 9 & 0,82 & 0,16 & 0,01 \\
Correspondance oral/écrit & 4 & 0,71 & 0,26 & 0,02 \\
Dénombrement & 4 & 0,86 & 0,20 & 0,02 \\
\hline Épreuve de mathématiques & 21 & 0,55 & 0,21 & 0,02 \\
Épreuve de français & 21 & 0,63 & 0,22 & 0,02 \\
\hline
\end{tabular}




\section{Pratiques éducatives familiales relatives à la préscolarisation}

Les pratiques éducatives familiales ont été opérationnalisées à partir du questionnaire de Tazouti et al. (2011). Il permet de recueillir des informations sur les pratiques éducatives des parents autour de la lecture, de l'écriture et du calcul. Une analyse factorielle confirmatoire concernant des items contenus dans le questionnaire a permis de mettre en évidence deux facteurs ( $c f$. tableau 2). Le premier sature les items 1 à 4 et renvoie à des « activités autour de l'enseignement formel de la lecture, de l'écriture et du calcul ». Le second sature les items 5 à 9 et correspond aux «activités non formelles autour du livre ». L'adéquation des données au modèle est satisfaisante puisque tous les indices d'adéquation ont des valeurs inférieures à celles considérées habituellement comme acceptables $\left(\chi^{2} \sim=32,86\right.$ pour 26 degrés de liberté ; $\mathrm{p}=0,17$; RMSEA (résidus) $=0,04$ ). Les coefficients de consistance interne (alpha de Cronbach) des items composant chacun de ces deux facteurs sont respectivement 0,70 et 0,50 .

\section{Tableau 2.}

Analyse factorielle confirmatoire sur les items relatifs aux pratiques éducatives familiales autour de la lecture, de l'écriture et du calcul en GS.

\begin{tabular}{|c|c|c|}
\hline & \multicolumn{2}{|c|}{ Facteur } \\
\hline & 1 & 2 \\
\hline $\begin{array}{l}\text { 1. Essayez-vous (ou un membre de votre famille) } \\
\text { d'apprendre à lire à votre enfant? }\end{array}$ & 0,84 & \\
\hline $\begin{array}{l}\text { 2. Essayez-vous (ou un membre de votre famille) } \\
\text { d'apprendre à écrire à votre enfant? }\end{array}$ & 0,66 & \\
\hline $\begin{array}{l}\text { 3. Utilisez-vous d'autres supports que le livre pour } \\
\text { apprendre à lire à votre enfant? }\end{array}$ & 0,42 & \\
\hline $\begin{array}{l}\text { 4. Essayez-vous (ou un membre de votre famille) } \\
\text { d'apprendre à compter à votre enfant? }\end{array}$ & 0,55 & \\
\hline 5. Votre enfant possède-t-il des livres à la maison? & & 0,37 \\
\hline $\begin{array}{l}\text { 6. Lisez-vous (ou un membre de votre famille) des livres à } \\
\text { votre enfant? }\end{array}$ & & 0,29 \\
\hline 7. Votre enfant est-il abonné à une revue ? & & 0,26 \\
\hline 8. Accompagnez-vous votre enfant à la bibliothèque? & & 0,69 \\
\hline 9. Achetez-vous des livres à votre enfant? & & 0,32 \\
\hline Alpha de Cronbach & 0,70 & 0,50 \\
\hline
\end{tabular}

\section{RÉSULTATS}

Dans un premier temps, nous analysons par des modèles de régression multiple les liens entre les caractéristiques individuelles et familiales des enfants (sexe, appartenance sociale, nationalité, pratiques éducatives familiales...) et les apprentissages premiers en GS. Dans un second temps, nous nous intéressons aux effets des apprentissages premiers en GS et des pratiques éducatives familiales sur les performances scolaires des élèves en CE1. 
Liens entre les caractéristiques individuelles et familiales des enfants et les apprentissages premiers en GS

Les modèles de régression multiple (tableau 3) présentent l'effet des caractéristiques individuelles et familiales des enfants sur les neuf domaines des apprentissages premiers en fin de GS. Toutes choses égales par ailleurs, on constate que :

- Les filles et les garçons obtiennent des performances comparables dans sept des neufs domaines d'apprentissages testés. Cependant, dans deux domaines, la mémoire visuelle et la correspondance oral/écrit, les filles obtiennent de meilleures performances.

- L'indice économico-culturel de la famille a un impact sur les scores dans les différents domaines des apprentissages premiers à l'exception de la mémoire visuelle. Dans ces huit domaines, plus l'indice économico-culturel est élevé meilleures sont les performances des enfants.

- Les pratiques éducatives familiales non formelles autour du livre ont un impact sur les scores aux épreuves de discrimination auditive et de correspondance oral/écrit. Plus les parents orientent leurs pratiques éducatives vers des activités non formelles autour du livre meilleures sont les performances des enfants dans ces deux épreuves. En revanche, on n'observe pas d'effet positif des pratiques éducatives familiales orientées vers l'enseignement formel de la lecture, de l'écriture et du calcul; ces pratiques ont même un impact négatif sur le score à l'épreuve du rythme.

- On n'observe pas d'effet de la variable nationalité de l'enfant sur les scores aux épreuves testant les apprentissages premiers, sauf pour l'épreuve de correspondance oral/écrit pour laquelle les enfants de nationalité étrangère obtiennent un score inférieur aux scores des enfants autochtones.

\section{Effets des apprentissages premiers en GS} et des pratiques éducatives familiales sur les performances scolaires des élèves en CE1

Les modèles de régression multiple ( $c f$. tableau 4$)$ ont été estimés pour apprécier l'effet les variables de l'étude sur les performances de CE1 en mathématiques. Dans ces neuf modèles, le score de CE1 en mathématiques correspond à la variable dépendante que nous cherchons à expliquer par différentes variables liées soit aux caractéristiques individuelles des élèves (qui ont le statut de variables contrôles), soit aux pratiques éducatives familiales et aux scores de GS (qui sont les variables cibles de cette étude). Toutes choses égales par ailleurs, on constate que :

- En mathématiques, les garçons obtiennent de meilleures performances scolaires en CE1 que les filles.

- La variable nationalité de l'enfant n'a pas d'impact les performances en mathématiques de CE1. 


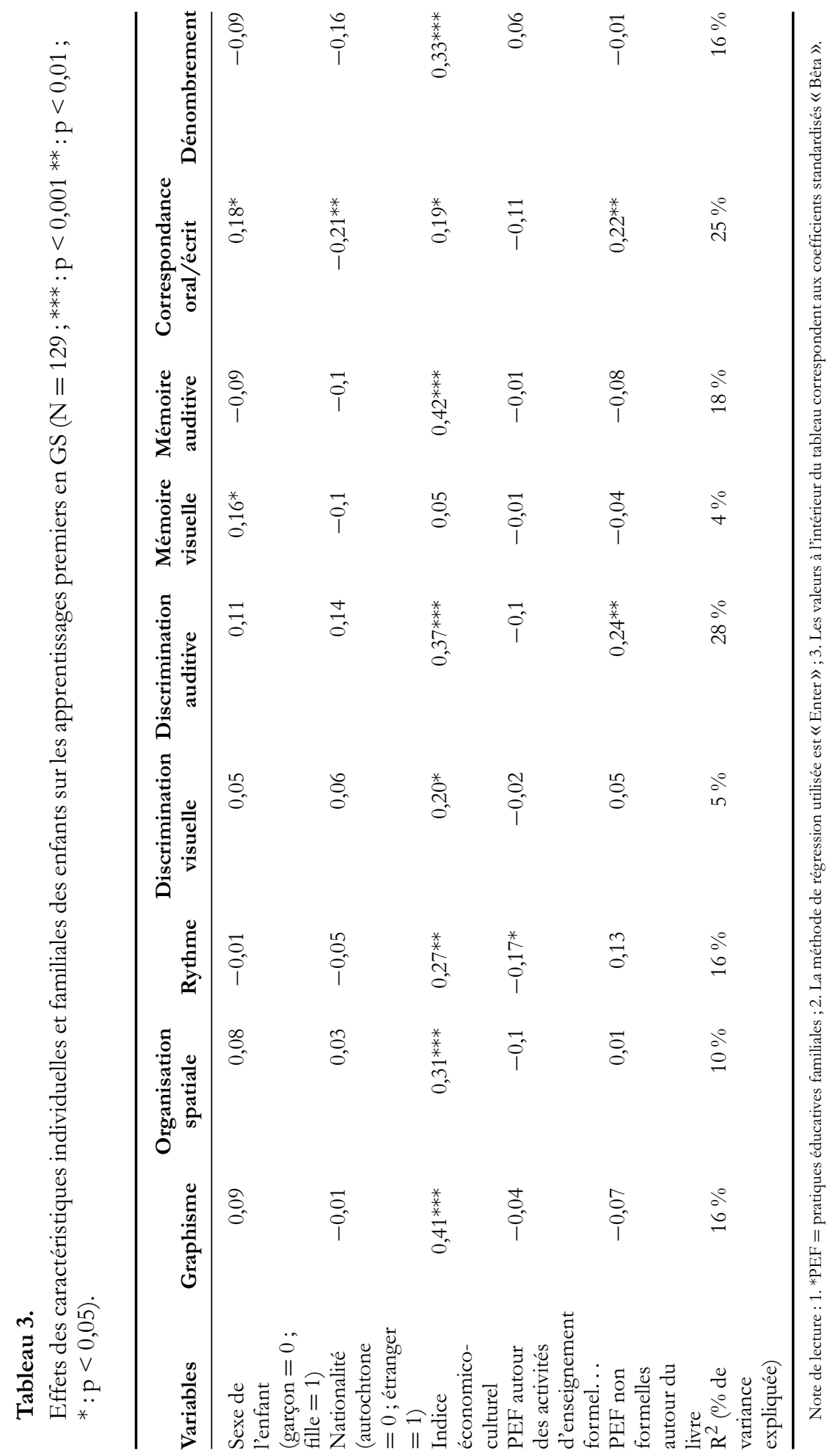




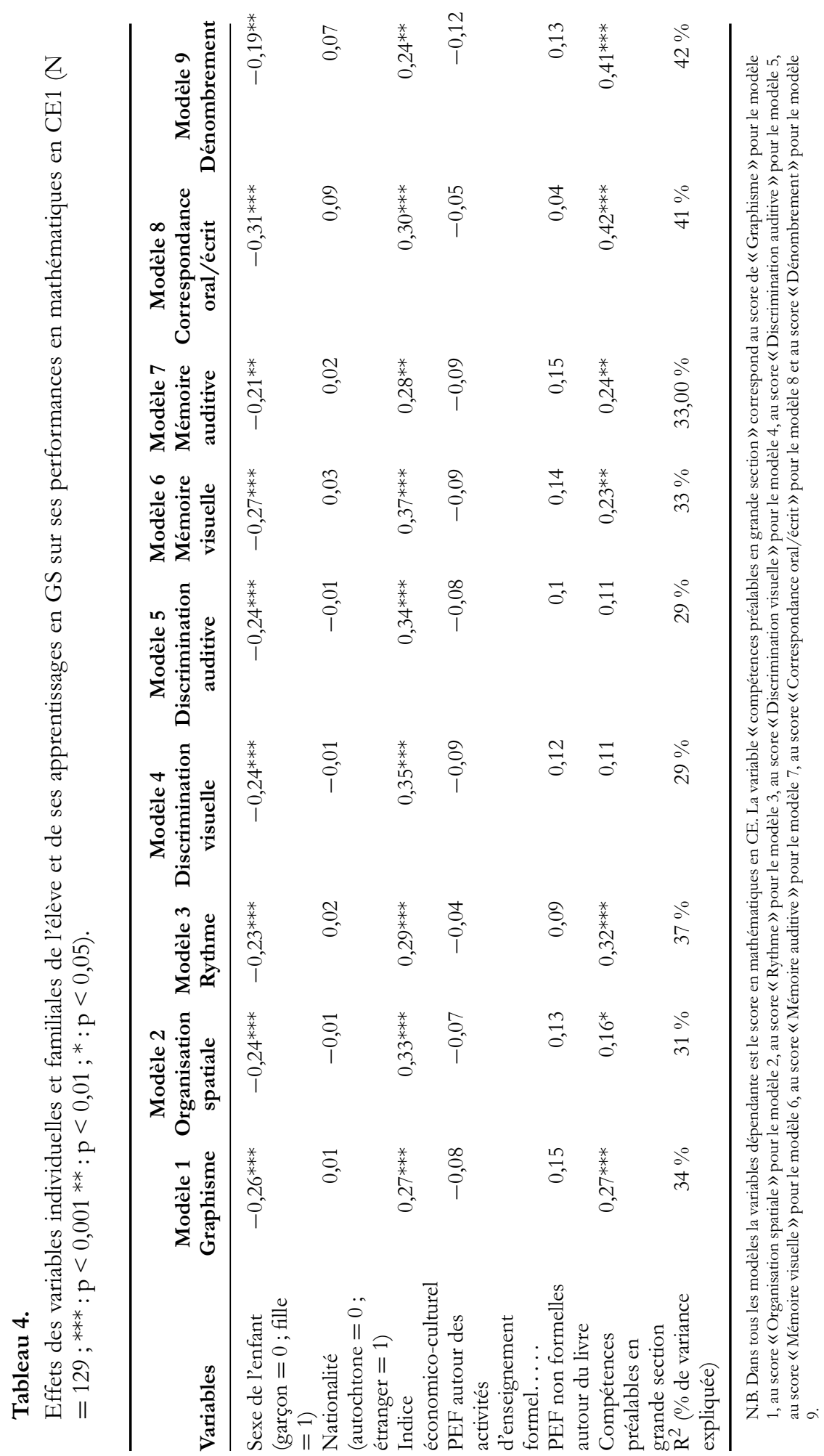


- L'appartenance sociale de la famille a un impact sur les performances en mathématiques en CE1.

- Les pratiques éducatives familiales de GS quelles qu'elles soient, formelles ou informelles, n'ont pas d'impact sur le score en mathématiques.

- Sept des neuf compétences préalables en grande section ont un impact positif sur le score en mathématiques de CE1. Dans l'ordre croissant du poids du coefficient de régression on distingue : l'organisation spatiale, la mémoire visuelle, la mémoire auditive, le graphisme, le rythme, le dénombrement et la correspondance oral-écrit.

De la même manière des modèles de régression multiple ( $c f$. tableau 5) ont été estimés pour apprécier l'effet des différentes variables de l'étude sur les performances de CE1 en français. Toutes choses égales par ailleurs, on constate que :

- Les filles et les garçons obtiennent des performances scolaires identiques.

- Comme en mathématiques, la variable nationalité de l'enfant n'a pas d'impact sur les performances de CE1 en français.

- L'appartenance sociale de la famille a un impact moins fort en français qu'en mathématiques. D'une part, on n'observe cet impact sur les performances en français que dans six modèles (modèles 11, 12, 13, 15, 17 et 18). D'autre part, lorsque l'effet de cette variable est significatif, le poids des coefficients de régression est inférieur à celui observé en mathématiques.

- Les pratiques éducatives familiales autour des activités d'enseignement formel de la lecture, de l'écriture et du calcul n'ont pas d'impact sur les performances de CE1 en français.

- Les pratiques éducatives familiales non formelles autour du livre en GS ont un impact sur les scores en français dans sept modèles (modèles 10, 11, 12, 13, 15, 16 et 18). Plus les parents orientent leurs pratiques éducatives vers des activités non formelles autour du livre en GS meilleures sont les performances des enfants en français en CE1.

- Huit des neuf compétences préalables en grande section ont un impact positif sur le score de CE1 en français. Dans l'ordre croissant du poids des coefficients de régression on distingue : l'organisation spatiale, le dénombrement, la discrimination visuelle, le graphisme, le rythme, la mémoire auditive, la correspondance oral-écrit et la discrimination auditive.

\section{DISCUSSION}

Notre étude qui a suivi une cohorte de 129 élèves depuis la grande section de maternelle jusqu'à la fin du CE1, apporte des résultats à trois niveaux différents. Tout d'abord, elle permet de conforter les résultats des recherches antérieures concernant les différences de performances des élèves à l'école maternelle. Elle met ensuite en évidence des liaisons fortes entre les acquisitions faites en GS et les acquisitions en français et en mathématiques faites en CE1. Elle met enfin en 


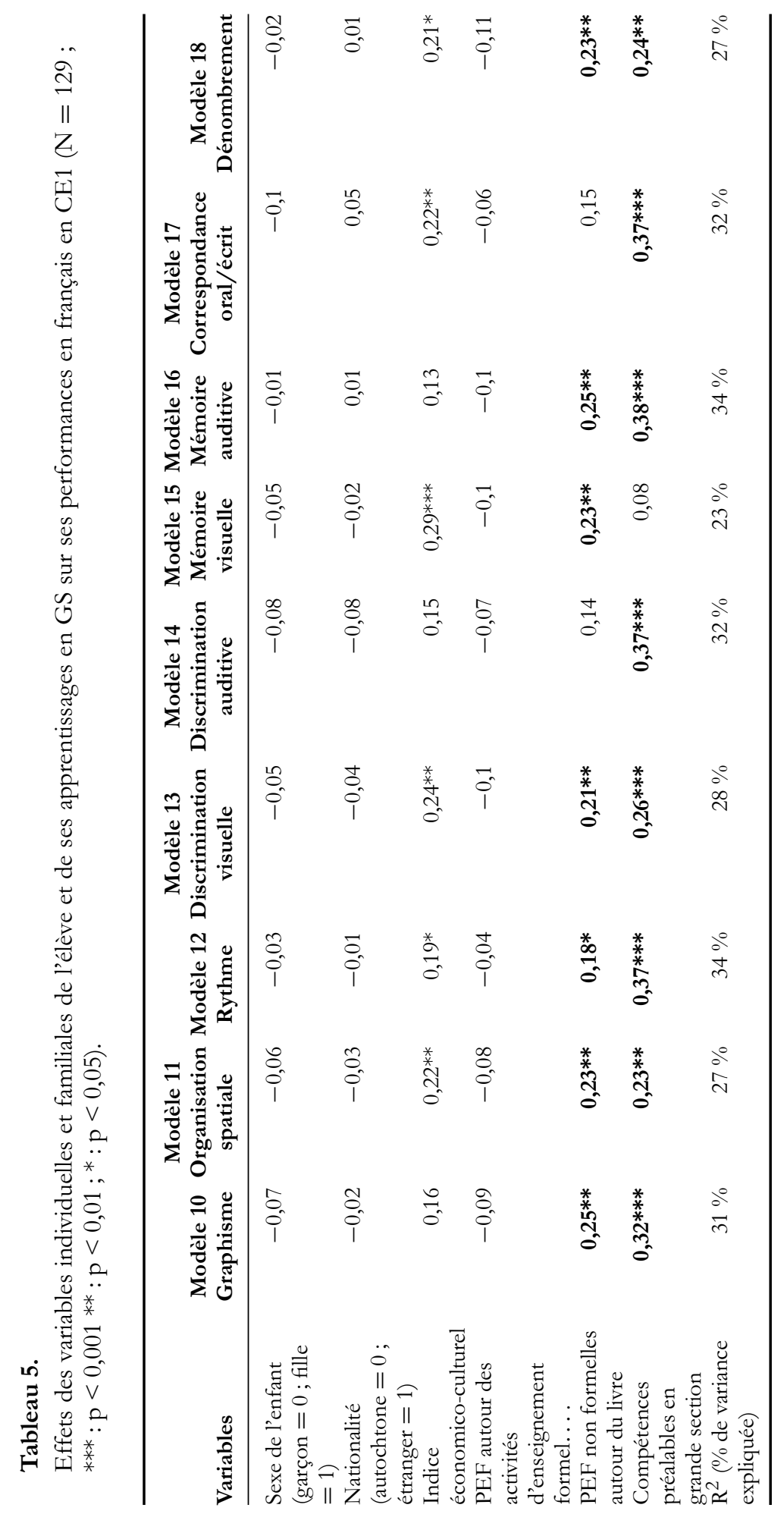


lumière des liens entre les pratiques éducatives familiales en lien avec la scolarité et les acquisitions de CE1.

\section{Des performances différenciées avant l'entrée au CP}

Nos résultats corroborent ceux des recherches antérieures qui montrent que, dès l'école maternelle, il existe des différences importantes de performances entre les élèves (Florin, Cosnefroy, \& Guimard, 2004). Ces différences de performances s'expliquent entre autres par l'origine sociale qui semble jouer un rôle important (Leroy-Audouin, 1993 ; Suchaut, 1996). En effet, d'après les travaux de Duncan et Seymour (2000) ou ceux de Raz et Bryant (1990) les enfants qui entrent à l'école avec un retard significatif dans différentes composantes de la littératie précoce, sont issus pour une grande majorité d'entre eux de milieux socio-économiques défavorisés.

En revanche, la variable sexe semble être moins discriminante à cet âge. On note un avantage significatif des filles, mais dans les champs de la mémoire visuelle et de la correspondance oral/écrit uniquement. La variable « sexe » n'affecte aucun des autres domaines évalués. Ces autres domaines, qui préparent en partie les futurs apprentissages de mathématiques et de français de l'école élémentaire, ne semblent donc pas être, à ce niveau de la scolarité, des domaines de prédilection de l'un ou l'autre sexe.

Concernant les pratiques familiales autour de la préscolarisation, seules les pratiques non formelles autour du livre ont un impact sur les scores aux épreuves de discrimination auditive et de correspondance oral/écrit. Ces résultats vont dans le sens de ceux précédemment mis en évidence au niveau de l'école maternelle (Tazouti et al., 2011)

\section{Des liens entre acquisitions de GS et acquisitions en CE1}

Concernant les liens entre les acquisitions de GS et les acquisitions de CE1 en français et en mathématiques, notre travail apporte un éclairage complémentaire aux travaux précédents centrés sur le caractère prédictif des acquisitions de CP sur les performances en fin d'école primaire (par ex. Caille \& Rosenwald, 2006). En effet, il corrobore les résultats observés dans d'autres systèmes éducatifs, tels ceux de La Paro et Pianta (2000), qui montrent des liens significatifs entre les apprentissages en maternelle et les acquisitions des élèves à l'issue des deux premières années de l'enseignement obligatoire.

Par ailleurs, cette étude permet d'identifier finement des compétences premières prédictives des performances de CE1 en français et en mathématiques. Dans le domaine du français, les compétences préalables liées à la discrimination auditive, à la correspondance oral/écrit et à la mémoire auditive s'avèrent liées aux performances de CE1. Ces compétences renvoient clairement à des habiletés liées à la conscience phonologique (par ex. capacités à identifier et à manipuler les syllabes et les phonèmes). Comme l'ont montré d'autres travaux (Bertelson, Morais, Alegria, \& Content, 1985 ; Snow, Tabors, Nicholson, \& Kurland, 1995), 
il existe une forte corrélation entre les mesures de conscience phonologique effectuées au préscolaire et le niveau en lecture atteint par les enfants en première année de primaire. Dans le champ des mathématiques, les compétences préalables liées à l'organisation spatiale, à la mémoire visuelle, au rythme et au dénombrement se révèlent liées aux performances de CE1. Ces compétences correspondent à des habiletés en numératie précoce (par ex. Hindman, Skibbe, Miller, \& Zimmerman, 2010).

\section{Des liens entre pratiques éducatives familiales et acquisitions en CE1}

Enfin, nous avons également montré que les pratiques familiales diversifiées et fonctionnelles dans le domaine de l'écrit (par ex. lecture de livres de jeunesse, fréquentation de bibliothèques...) sont plus corrélées positivement avec les performances des élèves que d'autres pratiques plus traditionnelles et formelles (par ex. enseignement des correspondances grapho-phonémiques). Ces résultats ont déjà été mis en évidence en GS par Prêteur et Louvet-Schmauss (1991). Notre étude montre de plus que ces pratiques familiales diversifiées et fonctionnelles dans le domaine de l'écrit ont un impact différé sur les acquisitions en français. En revanche, nos résultats ne montrent pas d'impact différé des pratiques familiales liées au nombre. Cependant, il est possible que cette absence d'effet soit liée à la construction de notre questionnaire qui accorde une place plus importante aux pratiques liées au langage écrit qu'à celles liées au nombre. La question de l'impact des pratiques familiales liées au nombre mériterait d'être approfondie.

\section{CONCLUSION}

Notre étude a permis de mettre en évidence au niveau de la maternelle, d'une part, des compétences qui s'avèrent prédictives des acquisitions de français et de mathématiques en CE1, et d'autre part, des pratiques familiales en lien avec la scolarité ayant un impact à long terme sur ces mêmes acquisitions.

Les résultats relatifs aux compétences prédictives des acquisitions de CE1 sont susceptibles de fournir des repères pour guider les enseignants dans le choix des activités à ne pas négliger de mener à l'école maternelle pour améliorer les apprentissages premiers des élèves et pour les préparer aux apprentissages ultérieurs. Notre travail ne permet évidemment pas de dire selon quelles modalités pédagogiques ces activités doivent être menées mais il met en évidence le caractère indispensable de ces activités. Par ailleurs, les résultats relatifs aux pratiques familiales, fournissent également des pistes pour aider les parents à accompagner la préscolarisation de leurs enfants en adoptant des pratiques éducatives non formelles autour du livre. Ils peuvent aussi apporter aux enseignants des informations pertinentes pouvant être portées à la connaissance des parents lors des rencontres parents-enseignants. 


\section{RÉFÉRENCES}

Applegate, B. (1986). A meta-analysis of the effects of day-care on development: Preliminary Findings. Paper presented at the annual meeting of the Mid-South Educational Research Association, Memphis, TN.

Bertelson, P., Morais, J., Alegria, J., \& Content, A. (1985). Phonetic analysis capacity and learning to read. Nature, 313, 73-74.

Bronfenbrenner, U. (2005). Making human beings human: Bioecological perspectives on human development. Thousand Oaks, CA: Sage Publications Ltd.

Caille, J. P., \& Rosenwald, F. (2006). Les inégalités de réussite à l'école élémentaire : Construction et évolution. In Insee, (dir.). France, portrait social (pp. 115-137). Paris $\sim$ : Insee.

Cèbe, S., \& Paour, J. L. (2000). Effect of cognitive education in kindergarten on learning to read in primary grades. Journal of Cognitive Education and Psychology, 1(2), 177-200.

Collins, R. C. (1984). Head Start: A review of research with implications for practice in early childhood education. Paper presented at the Annual Meeting of the American Educational Research Association, New Orleans, LA.

Duncan, L. G., \& Seymour, P. H. (2000). Socio-economic differences in foundationlevel literacy. British Journal of Psychology, 91, 145-166.

Epstein, J. L. (2001). School, family, and community partnerships. Boulder, CO: Westview Publishers.

Fan, X., \& Chen, M. (2001). Parental involvement and students' academic achievement: A meta-analysis. Educational Psychology Review, 13(1), 1-22.

Florin, A., Cosnefroy, O., \& Guimard, P. (2004). Trimestre de naissance et parcours scolaires. Revue Européenne de Psychologie Appliquée, 54, 237-246.

Fantuzzo, J. F., McWayne, C., Perry, M. A., \& Childes, S. (2004). Multiple dimensions of family involvement and their relations to behavioral and learning competencies for urban, low-income children. School Psychology Review, 33(4), 467-480.

Fusaro, J. A. (1997). The effect of full-day kindergarten on student achievement: A meta-analysis. Child Study Journal, 27, 269-277.

Goldring, E. B., \& Presbrey, L. S. (1986) Evaluating preschool programs: A metaanalytic approach, Educational Evaluation and Policy Analysis, 8, 179-188.

Harrell, A. (1983). The effect of the Head Start Program on children's cognitive development. Preliminary report. Head Start evaluation, synthesis and utilization project. Washington, D.C ; Superintendent of Documents, U.S. Government Printing Office.

Hatcher, P., Hulme, C., \& Snowling, M. (2004). Explicit phoneme training combined with phonic reading instruction helps young children at risk of reading failure. Journal of Child Psychology and Psychiatry, 45, 338-358.

Hattie, J. (2009). Visible learning: a synthesis of over 800 meta-analyses relating to achievement. London and New York: Routledge.

HCE : Haut Conseil de l'Evaluation de l'Ecole (2007). Bilan des résultats de l'école : L'école primaire. Paris : Ministère de l'Education Nationale.

Hindman, A. H., Skibbe, L. E., Miller, A., \& Zimmerman, M. (2010). Ecological contexts and early learning: Contributions of child, family, and classroom factors 
during Head Start to literacy and mathematics growth through first grade. Early Childhood Research Quarterly, 25, 205-235.

Hoover-Dempsey, K. V., \& Sandler, H. M. (2005). The social context of parental involvement: A path to enhanced achievement. Nashville, TN: Vanderbilt University.

Hoover-Dempsey, K. V, Battiato, A. C., Walker, J.M.T., Reed, R. P., DeJong, J. M., \& Jones, K. P. (2001). Parental Involvement in Homework. Educational Psychologist, 36, 195-209.

Innocenti, M. S., \& White, K. R. (1993). Are more intensive early intervention programs more effective? A review of the literature. Exceptionality, 4, 31-50.

Izzo, C. V., Weissberg, R. P., Kasprow, W. J., \& Fendrich, M. (1999). A longitudinal assessment of teacher perceptions of parent involvement in children's education and school performance. American Journal of Community Psychology, 27(6), 817-839.

Jones, S. (2002). Effect of all-day kindergarten on student cognitive growth: A meta-analysis. Unpublished Ed.D., University of Kansas, KS.

Leroy-Audouin, C. (1993). L'école maternelle entre la diversité des élèves et la continuité éducative : du passage anticipé au cours préparatoire au cycle des apprentissages fondamentaux. Thèse de doctorat en Sciences de l'Education non publiée, Université de Bourgogne.

La Paro, K. M., \& Pianta, R .C. (2000) Predicting children's competence in the early school years: A meta-analytic review. Review of Educational Research, 70(4), 443-484.

Manz, P. H., Fantuzzo, J. W., \& Power, T. J. (2004). Multidimensional assessment of family involvement among urban elementary students. Journal of School Psychology, 42, 461-475.

McWayne, C., Hampton, V., Fantuzzo, J. W., Cohen, H. L., \& Sekino, Y. (2004). A multivariate examination of parent involvement and the social and academic competencies of urban kindergarten children. Psychology in the Schools, 41, 363-377.

MEN (2003). Évaluations nationales Grande Section de Maternelle. Paris : Ministère de l'Education Nationale, de l'Enseignement Supérieur et de la Recherche.

MEN (2003). Évaluation à l'entrée au CE2 et en sixième. Repères nationaux septembre 2002. Paris : Ministère de l'éducation nationale.

Morlaix, S., \& Suchaut, B. (2007). Evolution et structure des compétences des élèves à l'école élémentaire et au collège : une analyse empirique des évaluations nationales. Cabiers de l'Irédu, 68, 259 p.

Murray, B. A., Stahl, S. A., \& Ivey, M. G. (1996). Developing phoneme awareness through alphabet books. Reading and Writing, 8(4), 307-322.

Nelson, G., Westhues, A., \& MacLeod, J. (2003). A meta-analysis of longitudinal research on preschool prevention programs for children. Prevention and Treatment, 18, 1-35.

OCDE (2006). Starting Strong II: Early Childhood Education and Care. Organisation de coopération et de développement économiques. [En ligne]. http://www. oecd.org/document/63/0,2340,en_2649_34819_37416703_1_1_1_1,00.html. Page consultée le 4 juin 2012

Paour, J. L., Cèbe, S., \& Haywood, H. C. (2000). Learning to learn in preschool education: Effect on later school achievement. Journal of Cognitive Education and Psychology, 1(1), 3-25.

Prêteur, Y., \& Louvet-Schmauss, E. (1991). Conception éducatives parentales vis-à-vis de l'apprentissage de la lecture chez l'enfant d'âge préscolaire : Étude 
comparative selon deux systèmes socioculturels et politiques (RFA et France). Enfance, 45, 83-97.

Purcell-Gates, V. (1996). Stories, coupons, and the TV guide : relationships between home literacy experiences and emergent literacy knowledge. Reading Research Quarterly, 31(4), 406-428.

Ravard, J. C., \& Rabreau, J. (2005). Echelle d'évaluation des préalables pour les CP et Batterie analytique de lecture/orthographe. Paris : Édition ECPA.

Raz, I. S., \& Bryant, P. (1990). Social background, phonological awareness and children's reading. British Journal of Developmental Psychology, 8(3), 209-225.

Rosenzweig, C. J. (2000). A meta-analysis of parenting and school success: The role of parents in promoting students' academic performance. Unpublished Ph.D., Hofstra University, NY.

Snow, P. E., Tabors, P. O., Nicholson, P. A., \& Kurland, B. F. (1995). Oral language and early literacy skills in kindergarten and first grade children. Journal of Research in Childhood Education, 10(1), 37-48.

Suchaut, B. (1996). La gestion du temps à l'école primaire : diversité des pratiques et effets sur les acquisitions des élèves. L'Année de la Recherche en Éducation, 1, 123-153.

Tazouti, Y., Viriot-Goeldel, C., Matter, C., Geiger-Jaillet, A., Carol, R., \& Deviterne, D. (2011). French nursery schools and German kindergartens: Effects of individual and contextual variables on early learning. European Journal of Psychology of Education, 26, 199-213.

Whitehurst, G., \& Lonigan, C. (1998). Child development and emergent literacy. Child Development, 69(3), 848-872.

Whitehurst, G. J., \& Lonigan, C. J. (2001). Emergent Literacy: Development from Prereaders to Readers. In S. B. Neuman \& D. K. Dickensen (Eds.), Handbook of Early Literacy Research (pp. 11-29). New York: Guilford Press. 
Annexe 1.

Exemples d'items des différentes épreuves des compétences préalables aux apprentissages fondamentaux

\begin{tabular}{|l|l|l|}
\hline Graphisme & $\begin{array}{l}\text { Consignes } \\
\text { simplifiées }\end{array}$ \\
\hline $\begin{array}{l}\text { Organisation } \\
\text { spatiale éléphant } \\
\text { va boire. Avec } \\
\text { votre crayon, } \\
\text { vous allez tracer } \\
\text { la route quili doit } \\
\text { prendre. } \\
\text { Attention! Il ne } \\
\text { faut pas toucher } \\
\text { les bords du } \\
\text { chemin. }\end{array}$ \\
\hline $\begin{array}{l}\text { Discrimination } \\
\text { visuelle }\end{array}$
\end{tabular}




\begin{tabular}{|c|c|c|}
\hline & Exemples d'items & $\begin{array}{l}\text { Consignes } \\
\text { simplifiées }\end{array}$ \\
\hline $\begin{array}{l}\text { Discrimination } \\
\text { auditive }\end{array}$ & & $\begin{array}{l}\text { Ecoutez bien et } \\
\text { n'oubliez pas } \\
\text { d'entourer le } \\
\text { mot dans lequel } \\
\text { on n'entend pas } \\
\text { la même chose } \\
\text { au début que les } \\
\text { autres. }\end{array}$ \\
\hline $\begin{array}{l}\text { Correspondance } \\
\text { oral/écrit }\end{array}$ & 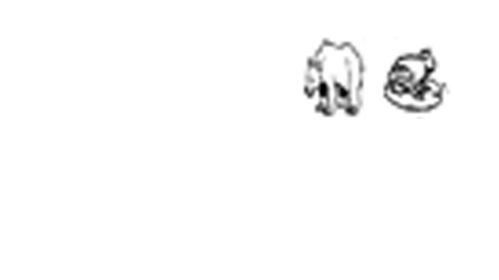 & $\begin{array}{l}\text { Vous allez } \\
\text { cherchez tout } \\
\text { seuls le mot le } \\
\text { plus long dans la } \\
\text { ligne. Je vous lis } \\
\text { deux fois les } \\
\text { mots. }\end{array}$ \\
\hline Dénombrement & 8 & $\begin{array}{l}\text { Complétez le sac } \\
\text { pour avoir six } \\
\text { billes. }\end{array}$ \\
\hline $\begin{array}{l}\text { Mémoire } \\
\text { visuelle }\end{array}$ & \multicolumn{2}{|c|}{$\begin{array}{c}\text { Les enfants regardent une image avec quatre dessins pendant } 30 \text { secondes. } \\
\text { Ensuite, ils doivent les retrouver dans une autre image comprenant une douzaine } \\
\text { d'éléments. }\end{array}$} \\
\hline $\begin{array}{l}\text { Mémoire } \\
\text { auditive }\end{array}$ & \multicolumn{2}{|c|}{$\begin{array}{l}\text { Les enfants écoutent une histoire. Ensuite, ils doivent entourer les animaux (ou } \\
\text { autres éléments) dont on avait parlé dans l'histoire. }\end{array}$} \\
\hline
\end{tabular}

